\title{
Filariose linfática em Belém, Estado do Pará, Norte do Brasil e a perspectiva de eliminação
}

\author{
Lymphatic filariasis in Belém, Pará State, North of Brazil \\ and the perspective of elimination
}

\author{
Gilberto Fontes ${ }^{1}$, Reinaldo F. Braun ${ }^{2}$, Habib Fraiha Neto ${ }^{3}$, João Batista F. Vieira ${ }^{4}$, Saturnino \\ S. Padilha ${ }^{2}$, Raimundo Cândido Rocha ${ }^{2}$ e Eliana M. Mauricio da Rocha ${ }^{1}$
}

\begin{abstract}
RESUMO
Com 0 objetivo de caracterizar a situação epidemiológica da filariose linfática em Belém-PA foram analisados dados dos inquéritos hemoscópicos de 1951 a 2003. As informações do período de 1951 a 1994 foram coletadas de relatórios disponibilizados pela Fundação Nacional de Saúde. Os dados de 1995 a 2003 foram obtidos através de inquéritos realizados em 62 bairros, dos oito distritos administrativos da cidade. Observou-se uma queda apreciável ao longo dos anos nos índices de microfilarêmicos. As percentagens de parasitados nas décadas de 1950, 1960, 1970, 1980 e 1990, foram respectivamente: 8,2\%, 2,6\%, 0,7\%, 0,16\% e 0,02\%. Em 2001, foi diagnosticado um único microfilarêmico, interrompendo uma série de dois anos sem registro de exames positivos na cidade. Em 2002 e 2003, inquéritos hemoscópicos e entomológicos foram realizados, simultaneamente, não sendo detectados indivíduos microfilarêmicos ou mosquitos infectados. Para manter essa tendência, medidas de vigilância devem ser observadas, a fim de detectar e tratar precocemente pacientes, para evitar o risco de ressurgimento dos focos, aparentemente já controlados.
\end{abstract}

Palavras-chaves: Epidemiologia. Filariose linfática em Belém. Wuchereria bancrofti. Inquéritos.

\begin{abstract}
The objective was to characterize the epidemiological situation of lymphatic filariasis in Belém, State of Pará. Hemoscopic data was analyzed from 1951 through 2003. Information for the period from 1951 to 1994 was collected from reports available from the National Health Foundation. Data from 1995 to 2003 was obtained through surveys carried out in 62 city sectors, within the eight administrative districts of the city. An appreciable drop in the microfilaraemic rates was observed over the years. The percentages of parasitized individuals in the decades of 1950, 1960, 1970, 1980 and 1990, were respectively: $8.2 \%, 2.6 \%, 0.7 \%, 0.16 \%$ and $0.02 \%$. In 2001 , a single microfilaraemic case was diagnosed, interrupting a series of two years without registering positive cases in the city. In 2002 and 2003, hemoscopic and entomological surveys were performed simultaneously revealing no microfilariae positive individuals, nor infected mosquitoes. To maintain this trend, surveillance measures must be conducted in order to detect and promptly treat patients, to prevent the risk of resurgence of a focus apparently now controlled.
\end{abstract}

Key-words: Epidemiology. Lymphatic filariasis in Belém. Wuchereria bancrofti. Surveys.

A filariose linfática ou bancroftose é uma enfermidade debilitante, com graves consequiências sociais e econômicas, causada no Brasil exclusivamente por helmintos da espécie Wuchereria bancrofti (Cobbold, 1877). 0 número de infectados no mundo é estimado em 120 milhões, distribuídos em 80 países de regiões tropicais ${ }^{25}$. É uma enfermidade negligenciada, sendo prevalente em populações de baixo nível sócio-econômico, carentes em saneamento e água tratada. 0 ser humano é 0 único hospedeiro vertebrado do parasito, albergando vermes adultos nos vasos linfáticos e microfilárias na circulação

1. Departamento de Patologia do Centro de Ciências Biológicas da Universidade Federal de Alagoas, Maceió, AL. 2. Secretaria Municipal de Saúde Belém, PA. 3. Núcleo de Medicina Tropical da Universidade Federal do Pará, Belém, PA. 4. Secretaria de Vigilância em Saúde do Ministério da Saúde.

Endereço para correspondência: Dr. Gilberto Fontes. Deptô de Patologia/Centro de Ciências Biológicas/UFAL. Pça Afrânio Jorge s/n, 57010-020 Maceió, AL, Brasil.

Tel: 5582 223-5613; Fax : 5582 221-2501

e-mail: gf@ fapeal.br

Recebido para publicação em 17/5/2004

Aceito em 16/11/2004 
sanguínea. Uma característica peculiar das microfilárias é a periodicidade noturna no sangue periférico dos hospedeiros, na maioria das regiões endêmicas ${ }^{12}$. 0 Culex quinquefasciatus (Say, 1823) é o principal transmissor onde ocorre a forma periódica noturna ${ }^{23}$.

Até 1950, muito pouco se sabia sobre a filariose linfática no Brasil, a não ser resultados de inquéritos hemoscópicos isolados para detecção da parasitose, os quais permitiram que cidades como Salvador (BA) e Belém (PA) fossem consideradas fo $\cos ^{11}$.

Em Belém (PA), um dos primeiros inquéritos para determinar a distribuição de W. bancrofti na população foi realizado entre 1942 e 1943, tendo sido encontrados, entre 5.000 examinados, 10,8\% microfilarêmicos ${ }^{3}$. Esses dados contribuíram para 0 Ministério da Saúde desencadear a Campanha Nacional contra a filariose linfática no Brasil, que incluiu a realização, entre 1951 e 1958, de inquéritos hemoscópicos e entomológicos. Ficou constatada a transmissão autóctone da filariose linfática em onze localidades no Brasil, descritas a seguir com as respectivas prevalências: São José da Ponta Grossa ( SC) , 14,5\%; Belém ( PA) , 9,8\%; Barra de Laguna ( SC) , 9,4\%; Recife (PE), 6,9\%; Castro Alves (BA), 5,9\%; Florianópolis (SC), 1,4\%; São Luis (MA), 0,6\%; Salvador (BA), 0,4\%; Maceió (AL), 0,3\%; Manaus (AM), 0,2\% e Porto Alegre (RS) , 0,1\% ${ }^{17}$. 0 tratamento das fontes de infecção, a partir de 1951, levou a uma apreciável redução nas taxas de prevalência. Na década de 1980, quase todos os focos descritos foram considerados extintos no Brasil, exceto, segundo 0 Ministério da Saúde, aqueles existentes em Belém (PA) e Recife (PE) (MS, 1985) ${ }^{15}$. 0 encontro de portadores autóctones de W. bancrofti, em Maceió (AL), em $1990^{6}$ e estudos subseqüentes ${ }^{9}$ ${ }^{18}$, mostraram que a capital alagoana se constituía um outro foco de transmissão ativa de filariose linfática no Brasil. Além disso, em Pernambuco, na atualidade, a parasitose também já foi detectada em cidades da região metropolitana de Recife, como Olinda, Jaboatão e Paulista ${ }^{1314}$.

As informações epidemiológicas da filariose linfática em Belém (PA) estão praticamente restritas aos órgãos de saúde locais, com a última publicação dos dados de prevalência feita em $1993^{10}$. Com 0 objetivo de se fazer uma avaliação da série histórica dos dados epidemiológicos da filariose linfática em Belém desde 1951, foram compilados os dados dos inquéritos hemoscópicos realizados a partir deste período até 2003. Estes dados foram analisados, a fim de se caracterizar a situação atual da bancroftose no único foco ainda considerado ativo no Norte do Brasil.

\section{MATERIAL E MÉTODOS}

Área geográfica. Área urbana do município de Belém, capital do Estado do Pará, dividida atualmente em oito distritos administrativos (D.A.) que englobam 71 bairros. Os D.A. são: 1) D.A. de Belém ( 8 bairros); 2) D.A. do Guamá (6 bairros); 3) D.A. da Sacramenta ( 7 bairros) ; 4) D.A. do Entroncamento ( 9 bairros) ; 5) D.A. do Bengüí ( 9 bairros); 6) D.A. de Icoaraci ( 9 bairros); 7) D.A. de Outeiro ( 4 bairros) ; 8) D.A. de Mosqueiro (19 bairros) (Figura 1).

População estudada. Na primeira fase do estudo, correspondente ao período de 1951 a 1994, foram colhidos dados referentes ao número de lâminas examinadas para pesquisa do parasito e número de lâminas positivas, obtidos dos relatórios de inquéritos na população de toda a cidade de Belém (PA), realizados anualmente e sucessivamente pelos seguintes órgãos: Serviço Nacional da Malária ( SNM), Departamento Nacional de Endemias Rurais (DNERu), Instituto Nacional de Endemias Rurais (INERu), Superintendência de Campanhas de Saúde Pública (SUCAM) e Fundação Nacional de Saúde ( FUNASA). Na segunda fase do estudo, de 1995 a 2003, os dados foram obtidos através de trabalho desenvolvido pela FUNASA e, posteriormente, pela Secretaria Municipal de Saúde de Belém (SMS), quando os inquéritos hemoscópicos passaram a ser apontados por distritos administrativos e bairros. Nesta segunda fase foram analisadas amostras de residentes de 62 bairros pertencentes aos oito distritos administrativos de Belém, incluindo todos aqueles considerados endêmicos no passado, e bairros selecionados aleatoriamente nas áreas onde nunca haviam sido detectados microfilarêmicos. A partir de 1995 a pesquisa foi realizada de acordo com a metodologia abaixo descrita.

Pesquisa de microfilárias. As colheitas de sangue foram realizadas casa a casa, por quarteirões escolhidos aleatoriamente. 0 consentimento foi obtido do responsável de cada família, após compromisso de tratamento dos parasitados. Com sangue obtido por punção capilar digital, era preparada uma lâmina de gota

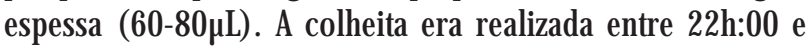
01h:00 devido à periodicidade noturna das microfilárias no sangue periférico do hospedeiro na regiã $0^{16}$. As gotas espessas de sangue, após desemoglobinizadas, eram fixadas com metanol coradas em eosina-Giemsa e examinadas ao microscópio óptico para pesquisa de microfilárias. Esta técnica é utilizada nos inquéritos hemoscópicos, por ser prática, rápida e econômica, constituindo-se referência para grandes inquérito $\mathrm{s}^{22}$.

Tratamento. Os microfilarêmicos detectados eram tratados com dietilcarbamazina na dose de $6 \mathrm{mg} / \mathrm{kg}$ de peso, durante 12 dias, como recomendado pela $\mathrm{MMS}^{24}$, com 0 acompanhamento de médicos do Sistema de Saúde local. Indivíduos que ainda apresentavam microfilárias no sangue após tratamento específico, eram tratados novamente.

Captura de insetos vetores e pesquisa de larvas de W. bancrofti. Em 2002 e 2003, mosquitos foram capturados dentro de residências escolhidas aleatoriamente em 57 bairros pertencentes a seis distritos administrativos, incluindo os bairros historicamente endêmicos para filariose linfática. Foram utilizados aspiradores de sucção para coleta de mosquitos adultos. Os mosquitos capturados foram identificados e fêmeas de C. quinquefasciatus foram dissecadas e examinadas com uso de microscópio para verificar a presença de larvas de W. bancrofti em diferentes estádios de desenvolvimento.

\section{RESULTAD0S}

Os resultados dos inquéritos hemoscópicos anuais, a partir de 1951, estão sumariados nas Tabelas 1 e 2. Entre 1951 e 1959, foram feitos pelo SNM e, posteriormente, pelo DNERu, 864.077 exames de gota espessa de sangue em moradores 


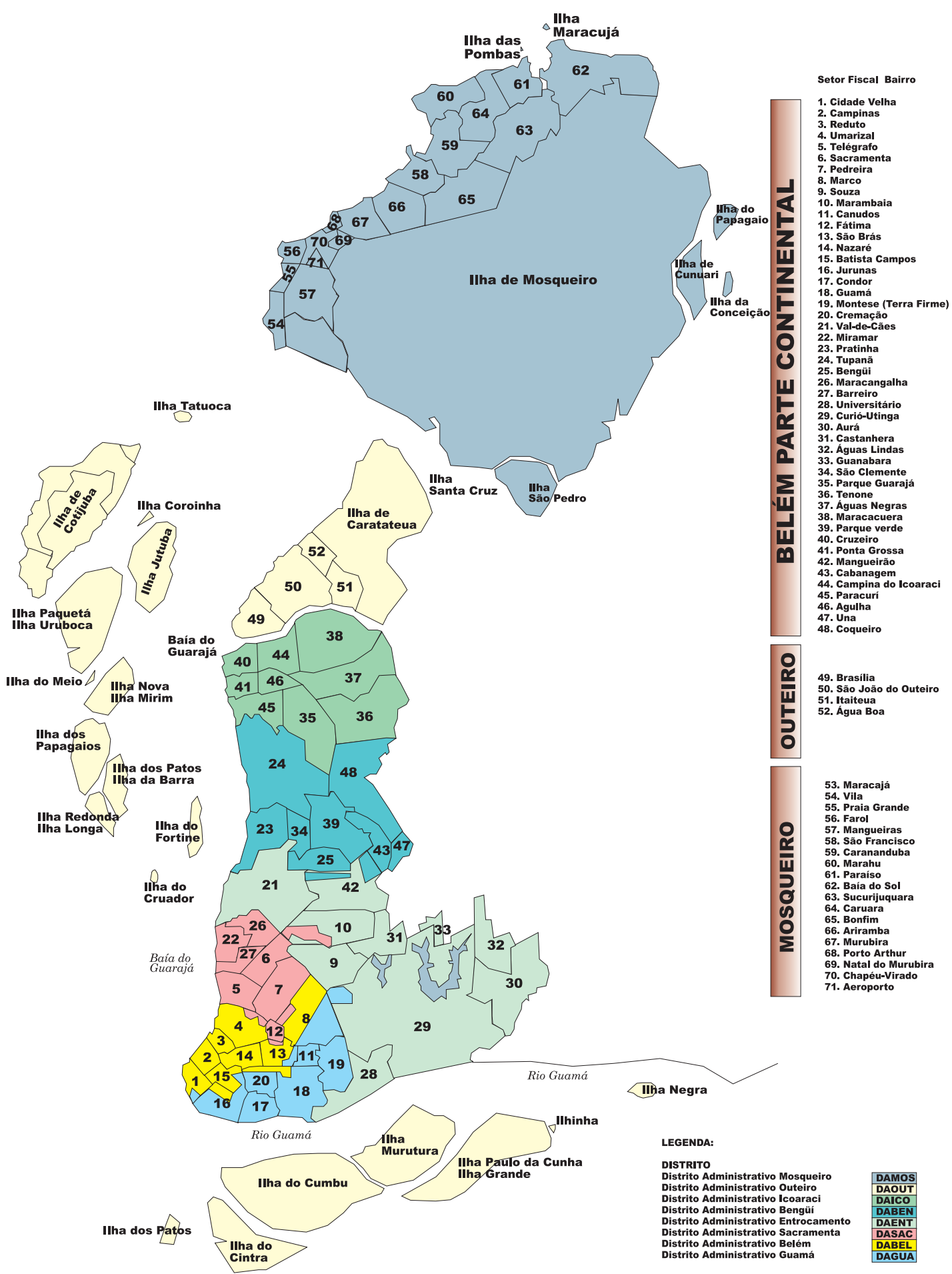

Figura 1 - Área Urbana do Município de Belém, PA, dividida em oito distritos administrativos com 71 bairros.

do município de Belém, tendo sido encontrados 70.637 (8,2\%) microfilarêmicos. De 1960 a 1969, ainda sob o controle do DNERu, foram realizados 2.450 .511 exames, com 62.863 (2,6\%) portadores de microfilárias detectados. Deve-se observar a proporção elevada de exames realizados a cada ano em relação à população total do município (Tabela 1). Em 1970, a SUCAM assume as campanhas de combate à filariose em Belém e realiza, nessa década, 3.684.208 exames por gota espessa de sangue na população, registrando 26.945 (0,7\%) microfilarêmicos. Na década seguinte, 1980 a 1989, foram realizados 3.470 .807 exames de sangue, dos quais $5.703(0,16 \%)$ apresentaram-se positivos. Entre 1990 e 1994 foram realizados 1.062 .945 exames, encontrando-se 180 (0,02\%) microfilarêmicos.

A Tabela 2 apresenta os resultados dos inquéritos hemoscópicos realizados por ano, a partir de 1995, na população geral da cidade, por bairros agrupados por distritos administrativos. Entre 1995 e 2003, foram examinados 1.116.525 indivíduos e encontrados 29 (0,0026\%) microfilarêmicos. Nesse período, a população de Belém variou de 1.203 .151 (1995) a 1.342.201 (2003). A Tabela 3 apresenta a percentagem de 
Tabela 1 - Inquéritos hemoscópicos realizados no período de 1951 a 1994, para detecção de microfilarêmicos por Wuchereria bancrofti na população geral do município de Belém, PA.

\begin{tabular}{|c|c|c|c|c|}
\hline \multirow[t]{2}{*}{ Ano } & \multirow{2}{*}{$\begin{array}{c}\text { Total da } \\
\text { População* }\end{array}$} & \multirow{2}{*}{$\begin{array}{l}\text { População } \\
\text { examinada }\end{array}$} & \multicolumn{2}{|c|}{ Microfilarêmicos } \\
\hline & & & $n^{0}$ & $\%$ \\
\hline 1951 & 288.519 & 8.588 & 845 & 9,8 \\
\hline 1952 & 300.060 & 31.625 & 6.306 & 19,9 \\
\hline 1953 & 312.062 & 49.417 & 6.721 & 13,6 \\
\hline 1954 & 324.545 & 43.824 & 4.148 & 9,5 \\
\hline 1955 & 337.526 & 45.501 & 5.595 & 12,3 \\
\hline 1956 & 351.027 & 67.021 & 5.953 & 8,9 \\
\hline 1957 & 365.069 & 163.579 & 13.826 & 8,5 \\
\hline 1958 & 379.671 & 215.579 & 14.231 & 6,6 \\
\hline 1959 & 394.858 & 238.943 & 13.012 & 5,4 \\
\hline 1960 & 412.170 & 274.763 & 10.713 & 3,9 \\
\hline 1961 & 428.656 & 227.016 & 9.737 & 4,3 \\
\hline 1962 & 445.803 & 242.183 & 8.110 & 3,3 \\
\hline 1963 & 463.635 & 308.835 & 9.110 & 2,9 \\
\hline 1964 & 482.180 & 278.678 & 6.828 & 2,5 \\
\hline 1965 & 495.000 & 264.850 & 4.566 & 1,7 \\
\hline 1966 & 521.526 & 243.597 & 4.379 & 1,8 \\
\hline 1967 & 542.387 & 222.649 & 4.353 & 2,0 \\
\hline 1968 & 564.082 & 195.175 & 2.471 & 1,3 \\
\hline 1969 & 586.646 & 192.765 & 2.596 & 1,3 \\
\hline 1970 & 642.514 & 339.882 & 3.862 & 1,1 \\
\hline 1971 & 668.214 & 291.508 & 2.540 & 0,9 \\
\hline 1972 & 694.943 & 316.835 & 2.753 & 0,9 \\
\hline 1973 & 722.740 & 230.809 & 2.283 & 1,0 \\
\hline 1974 & 751.650 & 216.412 & 2.027 & 0,9 \\
\hline 1975 & 771.665 & 186.593 & 3.238 & 1,7 \\
\hline 1976 & 812.985 & 345.853 & 2.668 & 0,8 \\
\hline 1977 & 845.504 & 670.174 & 3.657 & 0,5 \\
\hline 1978 & 879.324 & 547.781 & 2.184 & 0,4 \\
\hline 1979 & 914.947 & 538.361 & 1.733 & 0,3 \\
\hline 1980 & 949.545 & 553.782 & 1.723 & 0,3 \\
\hline 1981 & 978.031 & 423.226 & 1.114 & 0,3 \\
\hline 1982 & 1.007.371 & 376.425 & 1.003 & 0,3 \\
\hline 1983 & 1.047 .665 & 347.425 & 705 & 0,2 \\
\hline 1984 & 1.068 .720 & 285.031 & 332 & 0,1 \\
\hline 1985 & 1.116 .578 & 326.698 & 324 & 0,1 \\
\hline 1986 & 1.127 .743 & 310.458 & 216 & 0,1 \\
\hline 1987 & 1.139 .020 & 304.704 & 117 & 0,04 \\
\hline 1988 & 1.150 .411 & 310.858 & 90 & 0,003 \\
\hline 1989 & 1.161.915 & 232.200 & 79 & 0,03 \\
\hline 1990 & 1.167.841 & 225.674 & 71 & 0,03 \\
\hline 1991 & 1.179 .519 & 233.127 & 51 & 0,02 \\
\hline 1992 & 1.185 .416 & 205.428 & 31 & 0,015 \\
\hline 1993 & 1.191.343 & 226.542 & 14 & 0,006 \\
\hline 1994 & 1.197.300 & 172.174 & 13 & 0,007 \\
\hline
\end{tabular}

* Fonte: IBGE/PMB/SESMA/NSA examinados e número de microfilarêmicos detectados por distritos administrativos e bairros de Belém, a partir de 1995.

No inquérito entomológico foram visitados 6.759 imóveis, com captura de 48.927 espécimes fêmeas de C. quinquefasciatus, das quais 24.463 foram dissecadas, não se encontrando nenhuma larva de qualquer estádio de W. bancrofti.

\section{DISCUSSÃ0}

No final dos anos 1940 e início dos anos 1950 havia bairros em Belém como os de Guamá e Canudos, que juntos apresentavam percentagem de parasitados de $14,5 \%{ }^{5}$. Na década de 1950, os bairros que apresentaram os maiores índices de microfilarêmicos em Belém, eram Guamá, Terra Firme (atualmente Montese), Telégrafo, Sacramenta, Pedreira e ainda os distritos de Icoaraci e Mosqueiro ${ }^{5}$. Os dados de 1952 revelaram que 19,9\% dos examinados em Belém eram microfilarêmicos, além de que, com relativa freqüência encontravam-se pessoas já deformadas pela elefantíase ${ }^{19}$. Esses resultados acabaram por fazer do município de Belém a escolha lógica para campo da primeira tentativa de controle da filariose bancroftiana no Brasil ${ }^{11}$.

Nas décadas subseqüentes, observou-se decréscimo sistêmico no número de casos, que se manteve até 0 presente. Os microfilarêmicos encontrados a partir de 1995 estavam concentrados no distrito administrativo do Guamá e distrito administrativo da Sacramenta, que incluem os bairros de maior endemicidade na década de 1950 (Guamá, Terra Firme, Telégrafo, Sacramenta e Pedreira).

Entre 1960 e 1969, o número de exames realizados foi quase três vezes maior que 0 realizado na década anterior e a percentagem de parasitados foi reduzida de $8,2 \%$ para $2,6 \%$. A tendência à redução no número de microfilarêmicos no município de Belém continuou marcante, mostrando uma queda consistente e apreciável ao longo das décadas. A percentagem de parasitados nas décadas de 1970, 1980 e 1990, foi de respectivamente, $0,7 \%, 0,16 \%$ e $0,02 \%$.

Nos bairros historicamente endêmicos do município de Belém, como Guamá, Fátima, Pedreira, Sacramenta e Telégrafo, e no distrito de Icoaraci, que no passado também foi área endêmica, os últimos casos foram detectados entre os anos de 1996 e 1998.

Tabela 2 - Inquéritos hemoscópicos realizados no período de 1995 a 2003 para deteção de microfilarêmicos por Wuchereria bancrofti na população de diferentes bairros do município de Belém, PA.

\begin{tabular}{lccccrrr}
\hline Ano & $\begin{array}{c}\text { Distritos Administrativos } \\
\text { analisados* }\end{array}$ & $\begin{array}{c}\mathrm{N}^{p} \text { de bairros } \\
\text { analisados** }\end{array}$ & $\begin{array}{c}\text { População dos bairros } \\
\text { analisados }\end{array}$ & \multicolumn{2}{c}{$\begin{array}{c}\text { Amostra } \\
\text { examinada (\%) }\end{array}$} & \multicolumn{2}{c}{$\mathrm{N}^{\mathbf{m}}$} \\
\hline 1995 & $2,3,4$ & 06 & 331.276 & 226.796 & 68,5 & 6 & 0,002 \\
1996 & $2,3,6$ & 05 & 286.410 & 93.498 & 32,6 & 15 & 0,016 \\
1997 & 2,3 & 04 & 205.887 & 133.198 & 64,7 & 6 & 0,004 \\
1998 & 2,3 & 05 & 191.883 & 115.279 & 60,1 & 1 & 0,0009 \\
1999 & $4,5,6$ & 07 & 251.358 & 152.255 & 60,6 & 0 & 0,0 \\
2000 & $1,2,3,4$ & 06 & 205.081 & 132.388 & 64,6 & 0 & 0,0 \\
2001 & $1,2,3,6$ & 15 & 373.202 & 99.093 & 26,6 & 1 & 0,001 \\
2002 & $3,5,6,7,8$ & 21 & 547.412 & 92.463 & 16,9 & 0 & 0,0 \\
2003 & $2,4,5$ & 12 & 325.847 & 71.555 & 22,0 & 0 & 0,0 \\
\hline
\end{tabular}

* Distritos Administrativos ( D.A.): 1) D.A. de Belém; 2) D.A. do Guamá; 3) D.A. da Sacramenta; 4) D.A. do Entroncamento;

5) D.A. do Bengüí; 6) D.A. de Icoaraci; 7) D.A. de Outeiro; 8) D.A. de Mosqueiro.

** Alguns bairros foram pesquisados mais de uma vez em diferentes anos (vide Tabela 3) 
Tabela 3 - Percentagem de moradores examinados, por bairro e número de microfilarêmicos com Wuchereria bancrofti, 1995 a 2002, em Belém, PA.

\begin{tabular}{|c|c|c|c|c|c|c|c|c|c|c|}
\hline \multirow[t]{2}{*}{$\mathrm{DA}^{*}$} & \multirow[t]{2}{*}{ Bairro** } & \multicolumn{8}{|c|}{ Percentagem de examinados (\%) / número de parasitados encontrados } & \multirow[b]{2}{*}{2003} \\
\hline & & 1995 & 1996 & 1997 & 1998 & 1999 & 2000 & 2001 & 2002 & \\
\hline 1 & Cidade Velha & & & & & & $50,8 / 0$ & $9,8 / 0$ & & \\
\hline 2 & Canudos & & & & $83,9 / 0$ & & & $12,3 / 0$ & & $51,35 / 0$ \\
\hline 2 & Condor & & $20,4 / 0$ & & $44,3 / 0$ & & & $36,4 / 0$ & & \\
\hline 2 & Cremação & & $62,5 / 0$ & & & & $71,7 / 0$ & $8,8 / 0$ & & \\
\hline 2 & Guamá & $64,9 / 1$ & & $65,7 / 2$ & & & & $35,5 / 0$ & & $3,67 / 0$ \\
\hline 2 & Jurunas & & $24,2 / 1$ & & $58,6 / 0$ & & $59,0 / 0$ & & & \\
\hline 2 & T.Firme (Montese) & & & & $67,4 / 0$ & & & $12,6 / 0$ & & $32,10 / 0$ \\
\hline 3 & Barreiro & $75,7 / 0$ & & $63,7 / 0$ & & & $68,4 / 0$ & & $11,3 / 0$ & \\
\hline 3 & Fátima & & & & $61,6 / 1$ & & & $23,3 / 0$ & $32,6 / 0$ & \\
\hline 3 & Maracangalha & & & & & & & & $33,4 / 0$ & \\
\hline 3 & Pedreira & $63,8 / 3$ & $52,1 / 9$ & & & & & & $10,4 / 0$ & \\
\hline 3 & Sacramenta & $75,9 / 1$ & & $63,1 / 1$ & & & $68,5 / 0$ & & $13,8 / 0$ & \\
\hline 3 & Telégrafo & $75,6 / 1$ & & $63,7 / 3$ & & & & & $15,7 / 0$ & \\
\hline 4 & Águas Lindas & & & & & & & & & $48,17 / 0$ \\
\hline 4 & Aurá & & & & & & & & & $16,54 / 0$ \\
\hline 4 & Castanheira & & & & & $68,1 / 0$ & $66,5 / 0$ & & & $24,02 / 0$ \\
\hline 4 & Curió Utinga & & & & & & & & & $18,26 / 0$ \\
\hline 4 & Guanabara & & & & & & & & & $35,35 / 0$ \\
\hline 4 & Marambaia & $66,5 / 0$ & & & & $59,2 / 0$ & & & & $28,22 / 0$ \\
\hline 4 & Souza & & & & & & & & & $18,96 / 0$ \\
\hline 4 & Universitário & & & & & & & & & $12,48 / 0$ \\
\hline 5 & Bengui & & & & & $96,3 / 0$ & & & $10,4 / 0$ & \\
\hline 5 & Cabanagem & & & & & & & & $27,7 / 0$ & \\
\hline 5 & Coqueiro & & & & & & & & $38,4 / 0$ & $13,37 / 0$ \\
\hline 5 & Mangueirão & & & & & & & & $5,8 / 0$ & \\
\hline 5 & Parque Verde & & & & & & & & $6,2 / 0$ & \\
\hline 5 & Pratinha & & & & & $87,3 / 0$ & & & $22,5 / 0$ & \\
\hline 5 & São Clemente & & & & & & & & $6,8 / 0$ & \\
\hline 5 & Tapanã & & & & & $55,1 / 0$ & & & $16,4 / 0$ & \\
\hline 5 & Una & & & & & & & & $45,4 / 0$ & \\
\hline 6 & Icoaraci & & $17,9 / 5$ & & & & & $14,0 / 0$ & & \\
\hline 6 & Águas Negras & & & & & & & $30,1 / 0$ & $46,4 / 0$ & \\
\hline 6 & Agulha & & & & & & & $35,0 / 0$ & $30,8 / 0$ & \\
\hline 6 & Cruzeiro & & & & & & & $37,6 / 0$ & & \\
\hline 6 & Maracacuera & & & & & & & $23,1 / 1$ & & \\
\hline 6 & Paracuri & & & & & & & $54,6 / 0$ & & \\
\hline 6 & Parque Guajará & & & & & & & & $9,0 / 0$ & \\
\hline 6 & Ponta Grossa & & & & & & & $26,6 / 0$ & & \\
\hline 6 & Tenoné & & & & & $65,5 / 0$ & & & $5,6 / 0$ & \\
\hline 7 & Outeiro ( 4 bairros) & & & & & & & & $16,5 / 0$ & \\
\hline 8 & Mosqueiro (19 bairros) & & & & & & & & $24,9 / 0$ & \\
\hline
\end{tabular}

* Distritos Administrativos (D.A.) : 1) D.A. de Belém; 2) D.A. do Guamá; 3) D.A. da Sacramenta; 4) D.A. do Entroncamento; 5) D.A. do Bengüí; 6) D.A. de Icoaraci; 7) D.A. de Outeiro; 8) D.A. de Mosqueiro. ** Fonte: Secretaria Municipal de Saúde de Belém do Pará - Departamento de Vigilância à Saúde, 2003.

Em 2001, foi diagnosticado um único microfilarêmico, interrompendo uma série de dois anos sem registro de exames positivos na cidade de Belém. Este paciente apesar de procedente de Maracacuera, distrito de Icoaraci, residia na área há apenas oito meses, tempo inferior ao período pré-patente do parasito. 0 local provável de aquisição da infecção foi Icoaraci, local de origem do paciente e área endêmica no passado. Nenhum outro caso de microfilarêmico foi detectado em Maracacuera, inclusive entre familiares e vizinhos do parasitado detectado.

Os dados do inquérito entomológico realizado em 2002 e 2003 fortalecem a idéia da interrupção da transmissão da bancroftose no município de Belém. № início da década de 1950, foram iniciadas medidas de controle vetorial visando às formas larvais e adultas dos mosquitos vetores, através da destruição de criadouros e utilização de inseticidas de ação residual do tipo hexaclorobenzeno (BHC) e Dieldrin ${ }^{1120}$. Tais medidas foram abandonadas em 1956, devido à sua insustentabilidade 2 . Subseqüentemente e ao longo de cinco décadas, o controle da doença em Belém concentrou-se na identificação e no tratamento de microfilarêmicos ${ }^{2}$. Assim, o fator que provavelmente mais contribuiu para a queda progressiva e sistemática dos índices de microfilaremia em Belém, foi 0 tratamento específico com dietilcarbamazina, de milhares de parasitados detectados ${ }^{10}$. Situação semelhante ocorreu em Florianópolis, Barra de Laguna e São José da 
Ponta Grossa, focos no Estado de Santa Catarina, sul do Brasil, descritos na década de 1950. Aúnica medida realizada na época foi 0 tratamento dos parasitados detectados. Avaliação recentes nessa área mostrou a eliminação da bancroftose nestes focos do sul do Brasil $^{21}$. Em Maceió, Nordeste do Brasil, foco de transmissão ativa de bancroftose, a redução significativa dos índices de prevalência de microfilarêmicos e da infecção natural de mosquitos vetores, na atualidade, pode também ser atribuida ao tratamento especíico de microfilarêmicos com dietilcarbamazina, realizado nos últimos anos ${ }^{79}$.

Um outro fator que provavelmente contribuiu para o sucesso da campanha de eliminação da filariose linfática em Belém foi a manutenção de boas coberturas de tratamento, traduzidas em um número elevado de exames realizados a cada ano, em relação à população total do município. A busca ativa de casos, com imediata intervenção pelo tratamento, certamente foi 0 fator preponderante responsável pela eliminação das fontes de infecção na população. Ademais, extensas áreas das baixadas de Belém, onde no passado verificavam-se as maiores prevalências da filariose linfática, desde a segunda metade da década de 1990 estão submetidas a ações de manejo e saneamento ambiental de grandes proporções. Essas ações envolvem a drenagem de canais, remanejamento de populações de áreas alagadas, recuperação de áreas degradadas através de aterros, arborização e pavimentação, queprovavelmente também concorreram para a atual situação da filariose em Belém ${ }^{1}$. As ações de saneamento já atingem dezesseis bairros, correspondentes a uma área em torno de 40 $\mathrm{km}^{2}$, envolvendo uma população de mais de 600.000 habitantes ${ }^{1}$.

0 Plano de eliminação da filariose linfática, em andamento atualmente em Belém, inclui exames de gotas espessas de sangue da população geral, complementados por métodos mais sensíveis, como filtração de sangue em membrana de policarbonato, e métodos imunológicos, como pesquisa de antígenos através de imunocromatografia rápida ("ICT card test"), e ensaio imunoenzimático. Em paralelo são realizados inquéritos para determinação de infecção natural em mosquitos vetores ${ }^{1}$.

É objetivo da Organização Mundial de Saúde (OMS) e da Organização Pan Americana de Saúde (OPAS) , a eliminação da filariose linfática como problema de saúde pública no mundo até 2020, uma vez que esta enfermidade é incluída entre as doenças potencialmente eliminávei ${ }^{425}$. Baseado nos dados atuais, Belém, único foco de filariose linfática na região Norte do Brasil, poderá atingir essa meta antes da data programada. Para tal, devem ser mantidas as medidas devigilância, com inquéritos hemoscópicos e entomológicos, a fim de detectar e tratar precocemente pacientes, para evitar o risco de ressurgimento dos focos, aparentemente já controlados.

\section{AGRADECIMENTOS}

ADr. John Ehrenberg (OPAS/OMS, Washington DC) e Dr. Steven Ault (OPAS/OMS, Brasilia, DF), pela revisão e comentários. A Wendell A. Pinheiro de Almeida, pela adaptação da Figura.

\section{REFERÊNCIAS BIBLIOGRÁFICAS}

1. Braun RF. Plano de trabalho para a eliminação da filariose linfática. em Belém, Estado do Pará, Brasil. Secretaria Municipal de Saúde de Belém, Pará, impresso, 2002.

2. Braun RF. Eliminando a Filariose em Belém, Pará, Brasil. Boletim RPRG das Américas - Eliminação da Filariose linfática. Organização Pan-Americana da
Saúde/Organização Mundial da Saúde, OPAS/OMS. Brasília, Brasil, vol. 1, nº 2, p. 1 , agosto 2003.

3. Causey OR, Deane MP, Costa O, Deane LM. Studies on the incidence and transmission of filaria Wuchereria bancrofti in Belém, Brazil. American Journal of Hygiene 41: 143-149, 1945.

4. Center for Disease Control and Prevention (CDC). Recommendations of the International Task Force for disease eradication. Morbidity and Mortality Weekly Report 42: 1-38, 1993.

5. Deane LM, Damasceno RG. A filariose bancroftiana em Belém, Pará, segundo inquérito realizado em 1951. Revista Brasileira de Malariologia e Doenças Tropicais 4: 333-346, 1952.

6. Dreyer G, Medeiros Z, Béliz F, Vergetti G, Vergetti A, Café T, Fontes G. Autochthonous Wuchereria bancrofti microfilaremia in the city of Maceió-Alagoas-Brazil. Memórias do Instituto Oswaldo Cruz 86: 495-496, 1991.

7. Fontes G, Rocha EMM, Almeida WAP, Medeiros JS, Silva AS. Análise da infecção natural de insetos vetores de Wuchereria bancrofti em área endêmica definida: Ações para auxiliar a eliminação da Filariose linfática em Maceió/AL. Revista da Sociedade Brasileira de Medicina Tropical 35 ( supl I) : 218, 2002a.

8. Fontes G, Rocha EMM, Brito AC, Antunes CMF. Lymphatic Filariasis in Brazilian Urban Area (Maceió, Alagoas). Memórias do Instituto Oswaldo Cruz 93: 705-710, 1998.

9. Fontes G, Rocha EMM, Ferreira GER, Carvalho JMC, Ferreira CT. Programa de eliminação da Filariose linfática em Maceió/AL: Avaliação da prevalência de microfilarêmicos por Wuchereria bancrofti em áreas endêmicas definidas. Revista da Sociedade Brasileira de Medicina Tropical 35 ( supl I) : 222, 2002b.

10. Fraiha Neto H. Bancroftian filariasis in Belém, Pará State. Possibilities for eradication by introducing modern methods of control for Culex quinquefasciatus. Cadernos de Saúde Pública 9: 458-465, 1993.

11. Franco 0, Silva-Lima DM. Alguns aspectos das atividades contra a filariose bancroftiana no Brasil. Revista Brasileira de Malariologia e Doenças Tropicais 19: 73-89, 1967.

12. Hawking $F$, Jennings $T$, Louis FJ, Tuira $E$. The mechanisms which affect the periodic cycle of Pacific Wuchereria bancrofti microfilariae. Journal of Helminthology 55: 95-100, 1981.

13. Maciel A, Rocha A, Marzochi KBF, Medeiros Z, Carvalho AB, Regis L, Souza W, Lapa T, Furtado A. Epidemiological study of bancroftian filariasis in Recife, Northeastern Brazil. Memórias do Instituto Oswaldo Cruz 91: 449-455, 1996.

14. Medeiros Z, Dreyer G, Andrade L, Pires ML, Mendes J, Pimentel R. Wuchereria bancrofti microfilarial density of autochthonous cases and natural Culex infectivity rates in Northeast Brazil. Journal of Tropical Medicine and Hygiene 95: 214-217, 1992.

15. Ministério da Saúde. 0 controle das Endemias no Brasil (de 1979 a 1984). Brasília, DF, Superintendência de Campanhas de Saúde Pública, SUCAM, 1985.

16. Rachou RG. Da falta de correlação entre a periodicidade das microfilárias de Wuchereria bancrofti e o nascer e o ocaso do sol no norte e no sul do Brasil. Revista Brasileira de Malariologia e Doenças Tropicais 6: 395-405, 1954.

17. Rachou RG. Conceito e programa de profilaxia da filariose bancroftiana no Brasil. Revista Brasileira de Malariologia e Doenças Tropicais 12: 11-40, 1960.

18. Rocha EMM, Fontes G, Brito AC, Silva TRC, Medeiros Z, Antunes CMF. Filariose bancroftiana em áreas urbanas do estado de Alagoas, Nordeste do Brasil: Estudo em população geral. Revista da Sociedade Brasileira de Medicina Tropical 33: 545-551, 2000.

19. Scaff LM, Gueiros ZM. Prevalência e controle da filariose no Pará: estado atual. Revista Brasileira de Malariologia e Doenças Tropicais 19: 245-252, 1967.

20. Scaff LM, Gueiros ZM. Erradicação da filariose. Revista Brasileira de Malariologia e Doenças Tropicais 21: 603-613, 1969

21. Schlemper Jr. BR, Steindel M, Grisard EC, Carvalho-Pinto CJ, Bernardini OJ, Castilho CV, Rosa G, Kilian S, Guarnieri AA, Rocha A, Medeiros Z, Ferreira Neto JA. Elimination of bancroftian filariasis (Wuchereria bancrofti) in Santa Catarina state, Brazil. Tropical Medicine and International Health 5: 848-854, 2000.

22. Southgate BA. Recent advances in the epidemiology and control of filarial infections including entomological aspects of transmission. Transactions of the Royal Society of Tropical Medicine and Hygiene 78: 19-28, 1984

23. White GB. Lymphatic Filariasis. WHO. Geographical distribution of arthropod-borne diseases and their principal vectors. WHO-Vector Biology and control division. p. 23-34, 1989.

24. World Health Organization - Lymphatic filariasis: the disease and its control. Fifth report of the WHO Expert Committee on Filariasis. Geneva. Technical reports series, n. 821, 1992.

25. World Health Organization - Preparing and Implementing a National Plan to Eliminate Lymphatic Filariasis: A guideline for Programme Managers. Technical reports series WHO/CDS/CPE/CEE/2000.15. Geneva, 2000. 